\title{
Synthetic cationic antimicrobial peptides bind with their hydrophobic parts to drug site II of human serum albumin
}

\author{
Annfrid Sivertsen ${ }^{1}$, Johan Isaksson ${ }^{2}$, Hanna-Kirsti S Leiros ${ }^{1}$, Johan Svenson ${ }^{3}$, John-Sigurd Svendsen ${ }^{2,3}$
} and Bjørn Olav Brandsdal ${ }^{1,4^{*}}$

\begin{abstract}
Background: Many biologically active compounds bind to plasma transport proteins, and this binding can be either advantageous or disadvantageous from a drug design perspective. Human serum albumin (HSA) is one of the most important transport proteins in the cardiovascular system due to its great binding capacity and high physiological concentration. HSA has a preference for accommodating neutral lipophilic and acidic drug-like ligands, but is also surprisingly able to bind positively charged peptides. Understanding of how short cationic antimicrobial peptides interact with human serum albumin is of importance for developing such compounds into the clinics.

Results: The binding of a selection of short synthetic cationic antimicrobial peptides (CAPs) to human albumin with binding affinities in the $\mu \mathrm{M}$ range is described. Competitive isothermal titration calorimetry (ITC) and NMR WaterLOGSY experiments mapped the binding site of the CAPs to the well-known drug site II within subdomain IIIA of HSA. Thermodynamic and structural analysis revealed that the binding is exclusively driven by interactions with the hydrophobic moieties of the peptides, and is independent of the cationic residues that are vital for antimicrobial activity. Both of the hydrophobic moieties comprising the peptides were detected to interact with drug site II by NMR saturation transfer difference (STD) group epitope mapping (GEM) and INPHARMA experiments. Molecular models of the complexes between the peptides and albumin were constructed using docking experiments, and support the binding hypothesis and confirm the overall binding affinities of the CAPs.

Conclusions: The biophysical and structural characterizations of albumin-peptide complexes reported here provide detailed insight into how albumin can bind short cationic peptides. The hydrophobic elements of the peptides studied here are responsible for the main interaction with HSA. We suggest that albumin binding should be taken into careful consideration in antimicrobial peptide studies, as the systemic distribution can be significantly affected by HSA interactions.
\end{abstract}

Keywords: Albumin binding, Drug site II, Isothermal titration calorimetry, Group epitope mapping, Molecular docking, NMR, Crystal structure

\footnotetext{
* Correspondence: bjorn-olav.brandsdal@uit.no

${ }^{1}$ The Norwegian Structural Biology Centre, Department of Chemistry, Faculty of Science and Technology, University of Tromsø, NO-9037 Tromsø, Norway

${ }^{4}$ Centre for Theoretical and Computational Chemistry, Department of

Chemistry, Faculty of Science and Technology, University of Tromsø,

NO-9037 Tromsø, Norway

Full list of author information is available at the end of the article
} 


\section{Background}

Human serum albumin (HSA) is the most abundant transport protein present in blood plasma with a normal physiological concentration of $0.6 \mathrm{mM}$. It is active as a monomer with 585 residues and a molecular weight of $66.5 \mathrm{kDa}$. HSA has a high overall binding capacity due to a number of diverse binding sites distributed over the whole protein, and binds numerous endogenous and exogenous compounds [1]. The two best characterized binding sites regarding ligand specificity and structural information are drug site I and drug site II located in subdomain IIA and IIIA respectively [2]. HSA is known to have a preference for accommodating neutral lipophilic and acidic drug-like ligands, which corresponds well with its main function as a transporter of fatty acids. Exceptions to this generalization exist, and basic residues have been observed as HSA ligands, although only a few experimental complexes have been published [1]. So far the experimental basic ligand HSA complexes comprise the drug site I fluorescence marker dansyl-L-arginine bound in drug site I, and the anaesthetic compound lidocaine located in a novel binding site $[3,4]$.

HSA acts as a negative acute-phase protein, and its physiological concentration may decrease by as much as a factor of two in a number of physiological and pathological conditions [5]. This fluctuation in HSA plasma level affects the equilibrium between the bound and free fractions of a compound that binds to the transporter protein, and may affect drug dosage strategies [6]. In cases where highly hydrophobic compounds bind to HSA, the overall solubility of the drug in plasma will increase. The increase of drug solubility in plasma is regarded as beneficial, but a high affinity toward HSA will require higher dosages and may be a disadvantage [7]. The dual role of HSA binding depends on compound properties and comparative affinity strengths. Drugs binding to HSA are also prone to alterations caused by allosteric modulations induced by additional drug and fatty acid binding [7]. Changes in concentration of endogenous and exogenous ligands in plasma may further induce release of bound drugs into the free state by competing for the same binding site and result in toxic plasma levels [7].

Antimicrobial peptides (AMPs) are a part of the innate immune system of mammals, insects and plants, and act as a first line of defence against harmful microorganisms [8-11]. Most AMPs share the common features of an overall positive charge and an amphiphilic tertiary structure with clusters of cationic and hydrophobic residues; however, on the sequence and the secondary structure level a broad diversity is observed. AMPs are often the products of a pre-proprotein cleavage that are rapidly and inexpensively produced as an immune response. There are several proposed mechanisms for bactericidal activity of AMPs with interaction and disruption of the bacterial cell membrane being the common trait $[12,13]$. These unspecific membrane mechanisms are in agreement with experimental observations such as the broad activity and the need for relatively high AMPs concentrations to kill microorganisms. AMPs with receptorrecognition mechanisms exist, but are distinguished by having higher activities and specificities than those that interact with the bacterial membrane [12].

The synthetic cationic antimicrobial peptides (CAPs) studied in this work have been developed based on truncation and systematic mutations of lactoferricin, a natural AMP found in milk [14-16]. The determined pharmacophore established that the minimum motif for antimicrobial activity was two cationic charges and two hydrophobic moieties, and these could be incorporated in sequences as short as di- and tri-peptides [16]. The structure-activity relationship (SAR) has been further studied by incorporation of synthetic hydrophobic moieties and variation of the basic residues [17-20]. Although the peptides are too small to obtain any specific secondary structure, molecular modelling experiments indicate that flexible amphipathic conformations are one of the key properties of CAPs [18]. We have previously found that CAPs bind to albumin in the low $\mu \mathrm{M}$ range, and when HSA was included in cell-based assays at physiological concentrations the minimal inhibitory concentration (MIC) of the CAPs was increased by an order of magnitude [21]. Albumin binding has also recently been reported for other AMPs [22]. Previous ADMET studies on CAPs have mainly focused on protease stability, however blood plasma stability and stability in the main metabolic compartments of the body have also been investigated [20,23-25].

Studies of synthesized lactoferricin as retro, inverso and retroinverso versions support a bacterial membranedependent mechanism by being unable to differentiate between any of the activities of the variants [26]. In other investigations incorporating full $\mathrm{D}$-amino acid variants and D-amino acids in the CAP sequences, the antimicrobial activity is still retained compared with the native $[16,24]$. It is highly unlikely that a receptor-recognition mechanism would be unaffected by any of these structural modifications. Also supporting the membranolytic hypothesis is the fast bacterial killing and reported MIC values in the $\mu \mathrm{M}$ range [24]. Molecular dynamic simulations (MD) and NMR liposome dispersion studies of CAPs in a membrane system provide a reasonable interpretation of possible membrane interaction and indicate cell lysis by the carpet mechanism [27]. The Kallenbach group has studied the effect of scaffold attachment and density enrichment of peptides containing arginine and tryptophan, and suggest that an increase in density enhances antimicrobial activity [28-30].

So far a large amount of work has been done to investigate the different aspects of CAPs, with the main 
objective to develop a novel class of antibiotics for clinical use. As our previous albumin binding study indicates, HSA reduces the fraction of CAP in plasma when present at physiological concentrations, and hence reduces the antimicrobial activity. This behaviour affects how the CAPs will be managed in the next step of peptide engineering and if it is feasible to aim for systemic distribution in plasma. The small and drug-like molecular structure of the CAPs facilitates in part the potential for further development into new drugs. Plasma protein interaction of drug-like molecules is not uncommon as it is the rule rather than the exception in most cases. Detailed knowledge of the HSA interaction of CAPs would be useful in developing these peptides for use in combating the rise of antibiotic resistant bacteria.

Herein we report the binding of a selection of CAPs to human albumin along with competitive binding experiments with reference ligands for HSA drug site I and II determined by isothermal titration calorimetry (ITC) and NMR water ligand observation with gradient spectroscopy (WaterLOGSY). In order to examine the interaction in more detail NMR experiments applying saturation transfer difference (STD) for group epitope mapping (GEM) and INPHARMA for signal transfer between reference ligand and CAPs were conducted. Molecular docking was also carried out to obtain a better understanding of the binding mechanism.

\section{Results and discussion}

We have previously established that several CAPs surprisingly interact with HSA and bind with low $\mu \mathrm{M}$ affinity [21]. However the interaction between the peptides and HSA has not been explored in more detail. In order to examine the binding of CAPs to HSA with respect to drug site I or site II, five peptides were selected consisting of three active CAPs and two inactive control peptides as shown in Figure 1. CAP 1 and CAP 5 were included as controls as they contained either the positively charged arginines or one of the hydrophobic moieties necessary for activity [16].

\section{Antimicrobial activity}

The active peptides CAP 2-4 have been reported elsewhere to have MIC values ranging from 1.3 to $83 \mu \mathrm{M}$ (Table 1) [21,23,24]. The MIC values of CAP 1 and CAP 5 were above the threshold for all of the bacterial strains tested, and they are therefore regarded to be inactive as antimicrobial agents (Table 1). The lack of activity observed for CAP 1 was assumed to be a consequence of its lack of hydrophobic moieties, thus it does not fulfil the required pharmacophore. The inactivity observed with CAP 5 can be ascribed to its lack of cationic residues, as it is being composed only of hydrophobic residues.

\section{Isothermal titration calorimetry}

The thermodynamic data for non-competitive ITC experiments are presented in Table 2, and were similar to
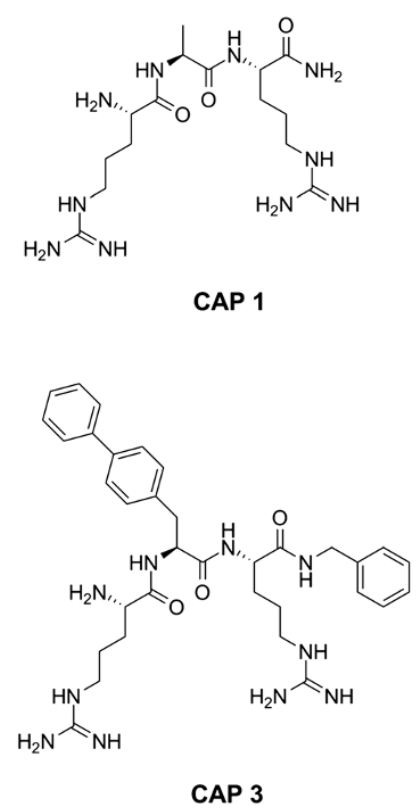

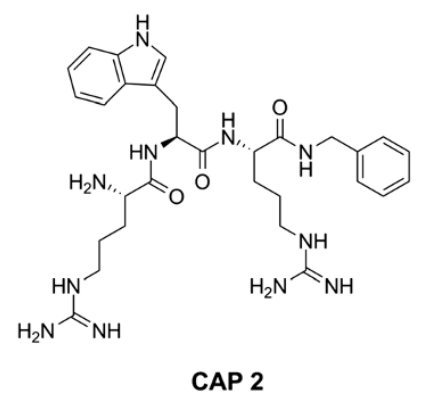<smiles>CN(C)c1cccc2c(O)cccc12</smiles><smiles>CC(O)CC(c1ccccc1)c1c(O)c2ccccc2oc1=O</smiles><smiles></smiles>

CAP 4

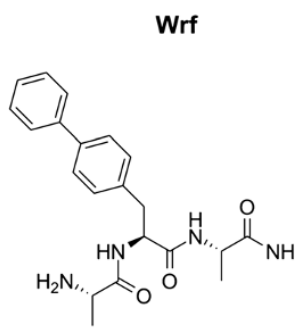

CAP 5

Figure 1 The molecular structure of CAPs and reference ligands used in this study. 
Table 1 Minimal inhibitory concentration values in $\mu \mathrm{M}$ towards selected bacteria

\begin{tabular}{|c|c|c|c|c|c|c|c|}
\hline Peptide & Ref. & S. aureus ${ }^{\mathrm{a}}$ & MRSA $^{b}$ & MRSE $^{c}$ & E. colid & P. aeruginosa ${ }^{\mathrm{e}}$ & $\mathrm{GISA}^{\mathrm{f}}$ \\
\hline CAP 1 & & $>499$ & $>499$ & - & $>499$ & - & - \\
\hline CAP 2 & [23] & 83 & 50 & 25 & - & - & - \\
\hline CAP 3 & {$[21,23]$} & $7.8,11$ & 11 & 3 & - & - & - \\
\hline CAP 4 & [24] & 3.2 & 3.2 & 1.3 & 9.7 & 6.5 & 3.2 \\
\hline CAP 5 & & $>523$ & $>523$ & - & $>523$ & - & - \\
\hline
\end{tabular}

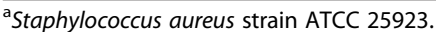

${ }^{\mathrm{b}}$ Methicillin resistant Staphylococcus aureus ATCC 33591.

${ }^{\mathrm{C}}$ Methicillin resistant Staphylococcus epidermidis ATCC 27626.

${ }^{\mathrm{d}}$ Escherichia coli ATCC 25922.

e Pseudomonas aeruginosa ATCC 27853.

${ }_{\mathrm{f}}^{\mathrm{G}}$ Glycopeptides intermediate-resistant Staphylococcus aureus CCUG 43315.

previously published binding data [21]. The control peptide CAP 1 did not bind to HSA, whereas the active peptides CAP 2-4 and the control peptide CAP 5 were found to interact with similar $K_{d}$ values in the low $\mu \mathrm{M}$ range. Raw data along with integrated heats and the binding isotherm model of a typical non-competitive titration experiment is presented in Figure 2 for CAP 4.

The binding constants $K_{d}$ for CAP 2-5 were in the same $\mu \mathrm{M}$ range $22-25 \mu \mathrm{M}(99-100 \mu \mathrm{M})$ (data obtained with MicroCal $\mathrm{iTC}_{200}$ are shown in parenthesis), indicating that all of the CAPs bind with the same affinity towards HSA (Table 2). The binding profiles show that there was both a favourable negative enthalpic and a positive entropic contribution to the free energy. For the known site I reference ligand Wrf (warfarin) the binding strength and thermodynamic profile were found to be similar compared with previous published studies, with our $\mathrm{K}_{\mathrm{d}} 9 \mu \mathrm{M}(28 \mu \mathrm{M})$ slightly higher compared with the previously reported values $2.9-3.8 \mu \mathrm{M}$ [31-33]. The binding affinity for drug site II reference ligand Dgly (dansylglycine) of $11 \mu \mathrm{M}(40 \mu \mathrm{M})$ is also slightly higher than the reported 1.7-3.2 published in other studies $[34,35]$. The corresponding free energies for Wrf and Dgly were respectively $-6.9(-6.2)$ and $-6.8(-6.0) \mathrm{kcal} / \mathrm{mol}$, with both favourable enthalpic and entropic contributions. By comparison, the CAPs binds with $K_{d}$ in the range $22-25 \mu \mathrm{M}$ (99-100 $\mu \mathrm{M})$, corresponding to free energies of -6.3 to $-6.4 \mathrm{kcal} / \mathrm{mol}(-5.5 \mathrm{kcal} / \mathrm{mol})$. When comparing the data obtained with the two instruments, the MicroCal $\mathrm{iTC}_{200}$ and the CSC 5300 Nano-Isothermal Titration Calorimeter III, the data values were observed to contain a variation. But there were consistency within the

Table 2 Thermodynamic ITC data for CAPs and reference ligands Wrf and Dgly

\begin{tabular}{|c|c|c|c|c|c|}
\hline Ligand & $\mathrm{K}_{\mathrm{d}}^{\mathrm{c}}{ }^{\dagger}$ & $\mathrm{n}^{\mathrm{d}, \neq}$ & $\Delta \mathrm{G}^{\mathrm{e}, \S}$ & $\Delta \mathrm{H}^{\mathrm{f}, \#}$ & $T \Delta S^{g, ~} \$$ \\
\hline CAP 1 & - & - & - & - & - \\
\hline CAP $2^{a}$ & $23 \pm 8$ & $0.67 \pm 0.07$ & $-6.32 \pm 0.18$ & $-3.7 \pm 0.5$ & $2.6 \pm 0.7$ \\
\hline CAP $3^{a}$ & $22 \pm 9(99 \pm 6)^{b}$ & $0.77 \pm 0.07(0.36 \pm 0.03)^{b}$ & $-6.36 \pm 0.19(-5.46 \pm 0.04)^{b}$ & $-2.8 \pm 0.4(-16.7 \pm 1.3)^{b}$ & $3.5 \pm 0.6(-11.2 \pm 1.4)^{b}$ \\
\hline CAP $4^{a}$ & $25 \pm 9$ & $0.57 \pm 0.08$ & $-6.28 \pm 0.19$ & $-5.2 \pm 1.0$ & $1.1 \pm 1.1$ \\
\hline CAP $5^{b}$ & $(100 \pm 13)$ & $(1.01 \pm 0.05)$ & $(-5.46 \pm 0.07)$ & $(-3.0 \pm 0.2)$ & $(2.5 \pm 0.3)$ \\
\hline Wrf $^{\mathrm{b}}$ (site I) & $9 \pm 4(28 \pm 4)$ & $1.15 \pm 0.04(1.02 \pm 0.02)$ & $-6.87 \pm 0.19(-6.21 \pm 0.07)$ & $-2.4 \pm 0.1(-3.3 \pm 0.1)$ & $4.4 \pm 0.3(2 . \pm 0)$. \\
\hline Dgly $^{b}$ (site II) & $11 \pm 4(40 \pm 2)$ & $1.08 \pm 0.05(1.16 \pm 0.01)$ & $-6.78 \pm 0.20(-6.00 \pm 0.02)$ & $-4.5 \pm 0.3(-5.5 \pm 0.1)$ & $2.3 \pm 0.5(0.5 \pm 0.1)$ \\
\hline
\end{tabular}

CSC 5300 Nano-Isothermal Titration Calorimeter III data, values obtained by MicroCal iTC200 presented in parenthesis. $\left(\mathrm{K}_{\mathrm{d}}\right.$ in $\mu \mathrm{M}, \Delta \mathrm{G}, \Delta \mathrm{H}$ and $\mathrm{T} \Delta \mathrm{S}$ in $\mathrm{kcal} / \mathrm{mol}$, $\mathrm{n}$ denotes the stoichiometric ratio).

a Three parallels.

${ }^{\mathrm{b}}$ Two parallels.

${ }^{c}$ Error limits taken as the average of the maximal difference between $K_{d}$ and calculated $K_{d} \pm$ error limits $95 \%$ confidence interval from $K_{a}$ CSC 5300 Nano-Isothermal Titration Calorimeter III, default error calculations based on least square fit and $X^{2}$ with $\mathrm{ITC}_{200}$

${ }^{d}$ Error limits are the averages of $\pm 95 \%$ confidence interval of the parallels CSC 5300 Nano-Isothermal Titration Calorimeter III, default error calculations based on least square fit and $\chi^{2}$ with $\mathrm{ITC}_{200}$.

${ }^{e}$ Error limits taken as the average of the maximal difference between $\Delta \mathrm{G}$ and calculated of $\Delta \mathrm{G} \pm$ error limits $95 \%$ confidence interval from $\mathrm{K}_{\mathrm{a}} \mathrm{CSC} 5300 \mathrm{Nano}-\mathrm{Isothermal}$ Titration Calorimeter III, default error calculations based on least square fit and $X^{2}$ with iTC 200 .

${ }^{f}$ Error limits are the averages of $\pm 95 \%$ confidence interval of the parallels CSC 5300 Nano-Isothermal Titration Calorimeter III, default error calculations based on least square fit and $X^{2}$ with $\mathrm{iTC}_{200}$.

${ }^{g}$ Error limits by cumulative addition of error limits of $\Delta \mathrm{G}$ and $\Delta \mathrm{H}$.

${ }^{\dagger}$ Deviation between parallels $<16 \%$, except Wrf with $39 \%$. ${ }^{\ddagger}$ Deviation between parallels $<21 \%$. ${ }^{\S}$ Deviation between parallels $<3 \%$. ${ }^{\#}$ Deviation between parallels $<12 \%$. \$Deviation between parallels $<20 \%$, except CAP 4 with $63 \%$. The symbol "-"denotes no measured binding. 


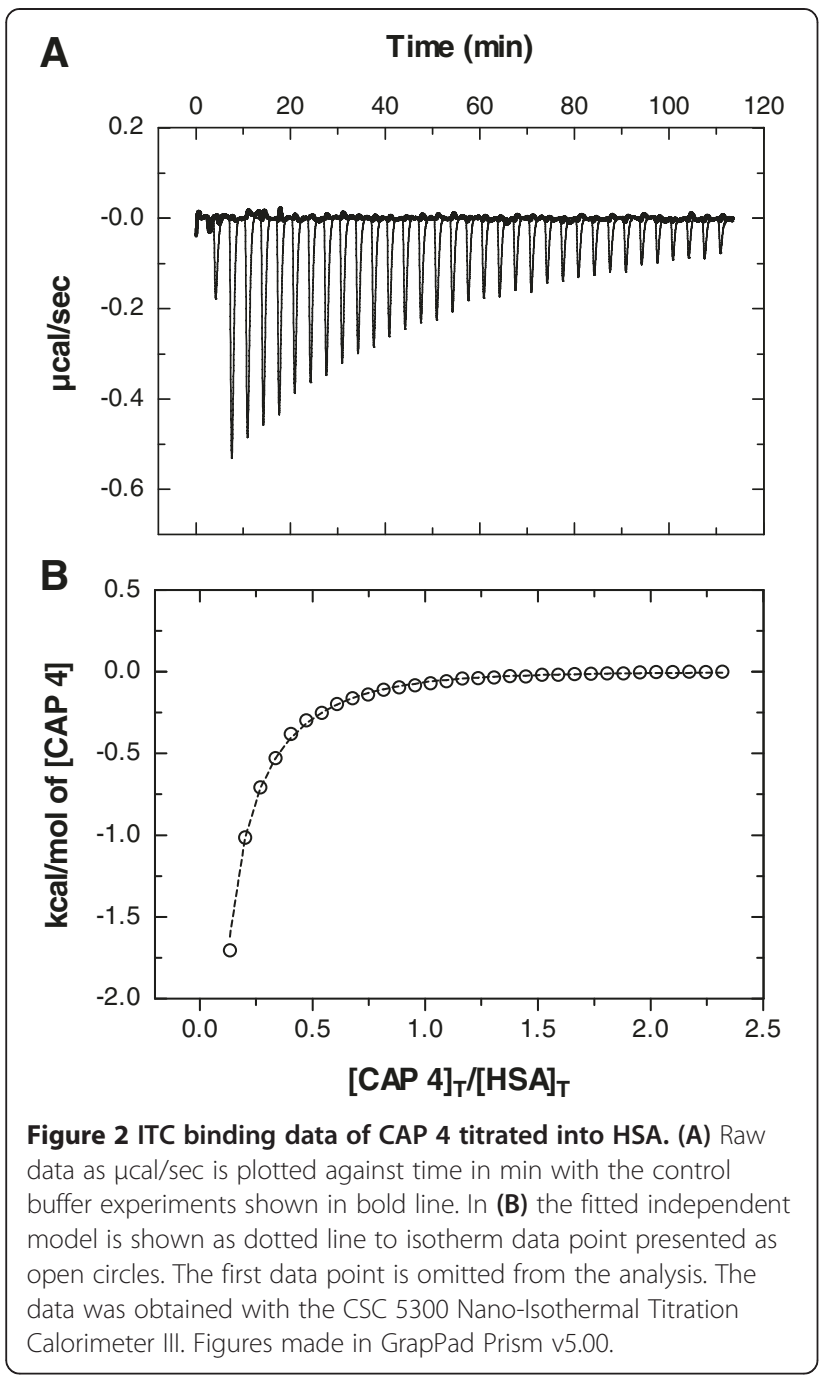

deviations in the binding affinities for the ligands determined with both instruments, hence if comparing the $\mathrm{K}_{\mathrm{d}}$ obtained with the MicroCal iTC $_{200}$ data the values showed 3-4 times lower affinities compared with the CSC 5300 Nano-Isothermal Titration Calorimeter III, e.g. $99 \mu \mathrm{M}$ compared with $22 \mu \mathrm{M}$ for CAP 3, $28 \mu \mathrm{M}$ and $9 \mu \mathrm{M}$ for Wrf and $40 \mu \mathrm{M}$ and $11 \mu \mathrm{M}$ for Dgly. Hence comparing the CAP 3 and CAP 5 binding in Table 2, CAP 5 was considered to bind in the same affinity range as the rest of the CAPs. The major difference in the comparison of the data between the instruments was the enthalpy-entropy profile of CAP 3 that changed to high favourable enthalpy but unfavourable entropy contribution with the MicroCal $\mathrm{iTC}_{200}$. The stoichiometry of the reference ligands Wrf and Dgly and the control peptide CAP 5 revealed a 1:1 ratio. Whereas the other peptides, CAP 2-4, the stoichiometry decreased to $0.6-0.8$ for the CSC 5300 NanoIsothermal Titration Calorimeter III instrument data, and even lower (0.4) for CAP 3 in the MicroCal $\mathrm{iTC}_{200}$ obtained data. The control buffer experiments did not show any significant heat of dilution signal for any of the experiments.

\section{Competitive binding in drug site II}

To further try to identify which of the numerous binding sites of HSA the CAPs were interacting with, competitive ITC experiments with the reference ligands Wrf and Dgly were conducted. Wrf only interacts with drug site I, whereas Dgly has a secondary binding site in drug site I in addition to its primary site in drug site II (Additional file 1: Figure S1). The competitive ITC results showed that the peptides competed with the drug site II reference ligand Dgly, and not with drug site I ligand Wrf. Competition was indicated by a significant decrease in the heat signals in all experiments where CAP 2, CAP 3, and CAP 4 were titrated into HSA incubated in 1:1 molar ratio with either Wrf or Dgly (data not shown). More pronounced was the decrease seen in the signals when HSA was incubated with 1:3 molar ratio either with Dgly or control peptide CAP 5, as shown in Figure 3 for CAP 3 titrations. The competitive effects for CAP 3 were equally strong for Dgly and CAP 5. Similar results were obtained for CAP 5 titrations into HSA incubated with Wrf or Dgly in molar ratio 1:3, data provided in Additional file 1: Figure S2. Control experiments reversing the titration order of the reference ligands and CAPs were performed for selected peptides and showed the same trends in competition pattern, data provided in Additional file 1: Figures S3 and S4. None of the control experiments with buffer in the cell indicated any significant ligand-ligand interactions in the competitive experiments. It should be noted that the competition experiments between Wrf and the peptides might indicate partial displacement of the ligand incubated with HSA. However, a more likely explanation is the alteration of the free ligand concentration due to peptide-Wrf interactions in solution. We see some interaction between Wrf and peptides in the NMR experiments (see below), which possibly lower the free concentration of the peptide in the ITC competition experiments. Nonetheless, the ITC data is clear on the main binding site as is evident from the Dgly competition experiments.

As a complementary technique, NMR was used to probe binding to HSA with WaterLOGSY experiments [36]. Weak binding could be confirmed for CAP 3 and CAP 5, but not for CAP 1 (Additional file 2: Figures S5 and S6). The CAP 3 peptide was selected as a representative CAP for competitive binding experiments in WaterLOGSY experiments versus Wrf and Dgly. The WaterLOGSY response to addition of competitive ligand shows a clear reduction in CAP 3 WaterLOGSY when titrated with Dgly but not with Wrf (Figure 4), thus confirming the ITC finding that CAP 3 and Dgly bind to the 

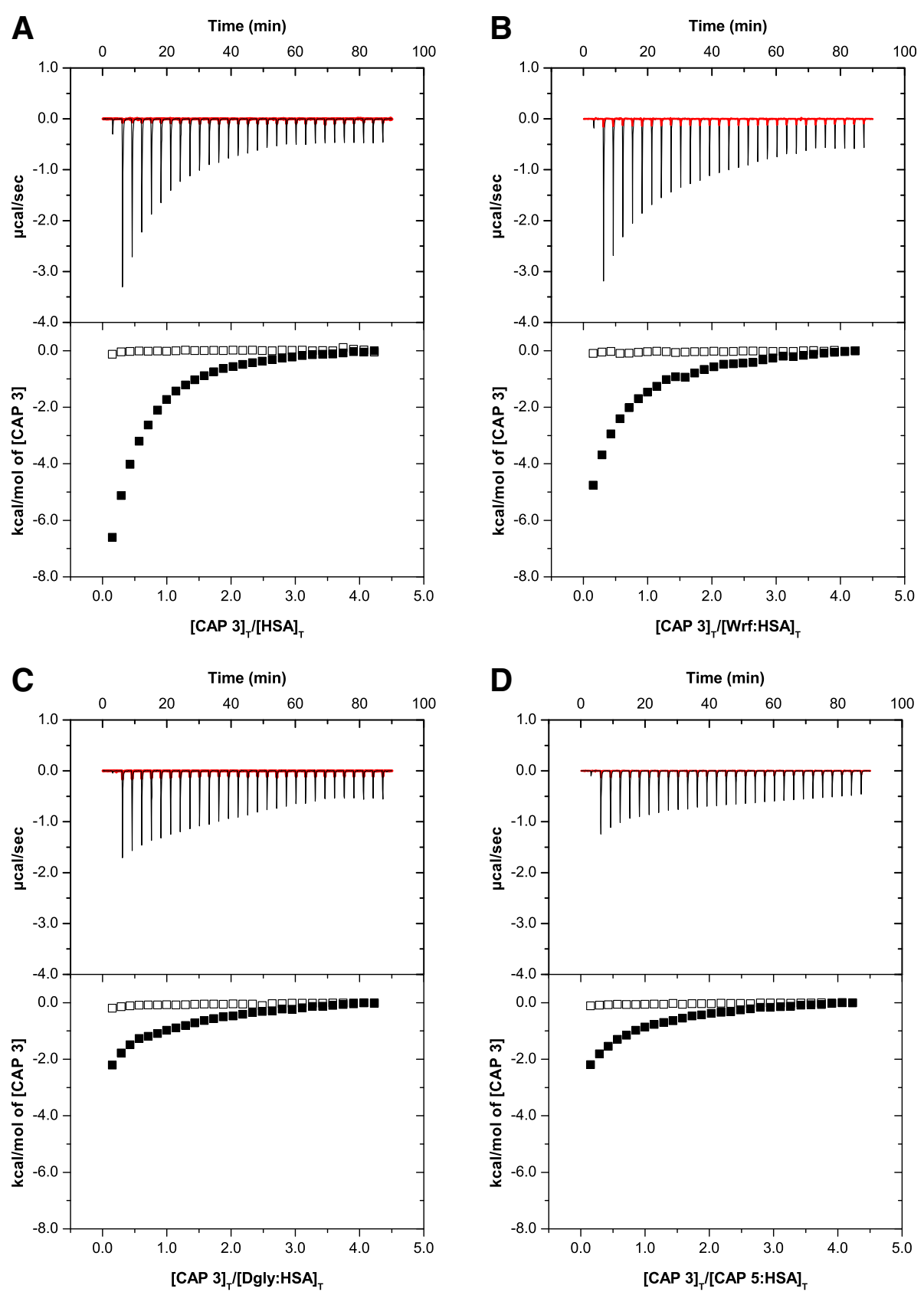

Figure 3 ITC raw data and integrated heats of CAP 3 titration into HSA. (A) CAP 3 titrated into HSA solution (reference data) and (B) competitive experiments of HSA incubated 1:3 molar ratio with drug site I reference ligand Wrf, (C) with drug site II reference ligand Dgly and (D) with control peptide CAP 5. Control buffer titration is shown in red line in the upper panel and open squares in the lower panel. (The molar ratio in the buffer control was set to the same as the protein ligand ratio merely for the purpose of interpretation). Data collected with MicroCal iTC 200 . Figures made in Origin ${ }^{\oplus} 7.0$.

same site on HSA. There were no indications of any direct interactions between Dgly and the studied CAPs before the addition of HSA to the sample. However, a weak direct interaction between CAP 3 and Wrf in solution was observed as weak direct NOE correlations (also inverting the NOE sign to negative) between the two ligands in trNOE experiments as well as a reduction in relaxation times for the biphenyl resonances of CAP 3 upon Wrf addition (data not shown).

The CAPs interact with drug site II with their hydrophobic moieties

The INPHARMA results of CAP 3 and Dgly show signal transfer between the biphenyl residue and the benzyl 


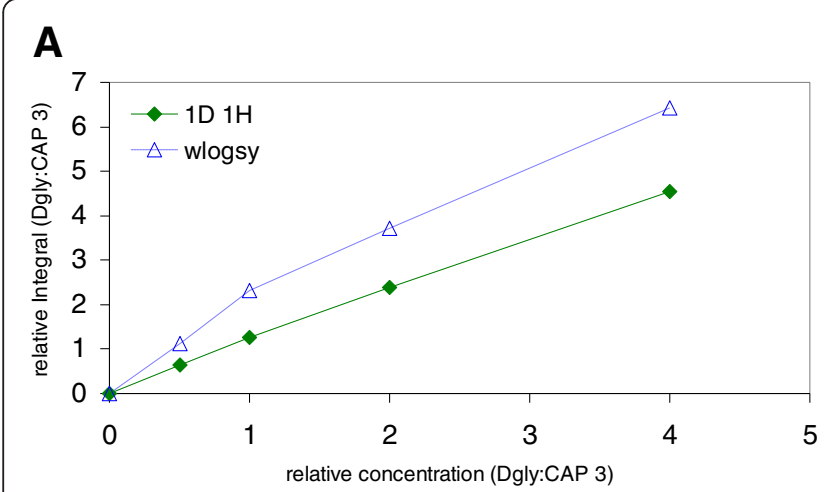

\section{B}

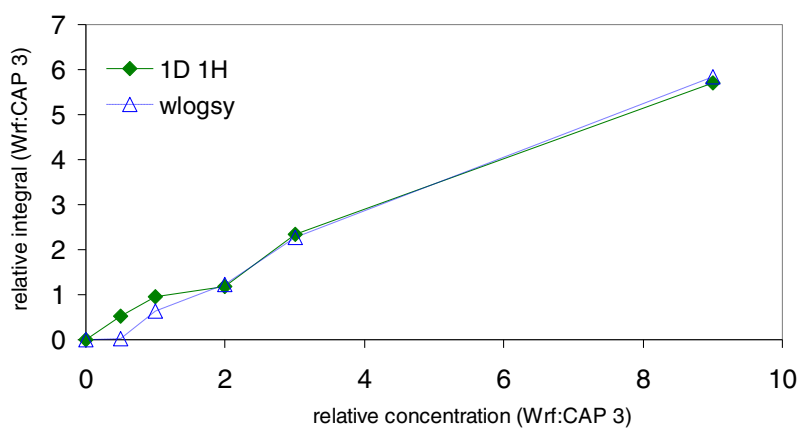

Figure 4 Competetive WaterLOGSY between CAP 3 and Dgly (A) and Wrf (B). (A) shows how Dgly perturbs 1:1 linear buildup of the WaterLOGSY effect which is proportional to protein binding, in CAP $\mathbf{3}$ relative to the concentration of added Dgly, whereas (B) shows that Wrf does not produce the same effect. Ligand:protein ratio was 20:1.

capping group of the peptide and the reference ligand (see Figure 5 and Table 3). This is best seen in the signal transferred from the tertiary amine methyl groups of Dgly and the hydrophobic moieties of CAP 3. The epitope mapping of Dgly is in agreement with the results reported in Lucas et al. showing that the strongest interaction is between the N-methyl groups as well as the glycine methylene group of Dgly and HSA [37]. It was clear from the data that the cationic arginines did not contribute to the interaction between CAP 3 and drug site II of albumin as no signal was transferred to these parts of the peptide.

The saturation transfer difference with group epitope mapping further confirmed the INPHARMA results. The arginines of CAP 3 were not excited by saturation of protein methyl groups, whereas the hydrophobic parts of the molecules clearly lit up as seen in Figure 6, indicating that they directly take part in interactions with the protein. For Dgly the whole molecule is excited which is in agreement with the INPHARMA results.

\section{Exploration of modelled binding modes}

Drug site II is located in subdomain IIIA of HSA, and has similarities to drug site I in subdomain IIA. Both of the sites are characterized by an apolar pocket and a basic polar patch at the binding site entrance, with a preference for aromatic drug-like ligands with a peripherally negative charge $[38,39]$. The main difference between the two sites is the packing environment, which enables drug site I to be larger with an enhanced flexibility compared to drug site II [38]. The two fatty acid binding sites (FA) 3 and 4 are also associated with drug site II. In FA3 the methylene tail of the fatty acid is bent into the apolar pocket of drug site II, and in the case of FA4 the carboxyl group of the fatty acid is hydrogen bonded with residues in the polar patch at the site entrance $[40,41]$. As the HSA ligand preferences for this site are aromatic and negatively charged small ligands, the experimentally determined binding of cationic peptides interacting with this site by both ITC and NMR was unanticipated. The comparably larger size and the multiple positive charges provided by two arginines and the protonated $\mathrm{N}$-terminal of the peptides were expected to exclude CAPs as drug site II ligands.

To further investigate the binding mechanism molecular docking was performed targeting drug site II of HSA. In general, the modelled docking poses and scores supported the experimental findings from both the ITC and NMR data. In all of the docking experiments, the reference drug site II ligand Dgly was docked in a position similar to that occupied by the ligand from original structure, and was located at the top of the docking score ranking list. The modelled conformations of Dgly were found to be virtually identical for all of the target structures, which were accommodated in the apolar pocket of site II. The docking mode was in agreement with the NMR INPHARMA and STD GEM interpretation of Dgly binding. The tertiary amine was buried in the inner hydrophobic part of the site, with the carboxy and sulphate group hydrogen bonding with the polar patch residues at the site entrance. Only docking poses of the CAP library that would compromise the volume occupied by Dgly were accepted, as the results from the competitive ITC data and the NMR WaterLOGSY indicate. The reference ligand Wrf for drug site I and the non-binding peptide CAP $\mathbf{1}$ were added as negative controls, and were generally given docking scores in the lower part of the ranking list.

The main difference between the docking targets was the conformation of the entrance residue Arg410. This residue is flexible and known to be a highly ligand inducible residue, which is also reflected by high $\mathrm{B}$ factors in the structure of $1 \mathrm{E} 78$ and 2BXF, or disordered as found for crystal structures 2XW1 and 2XVQ. Three different main conformations were observed, either pointing towards Glu492 as seen in the Prime built and minimized 


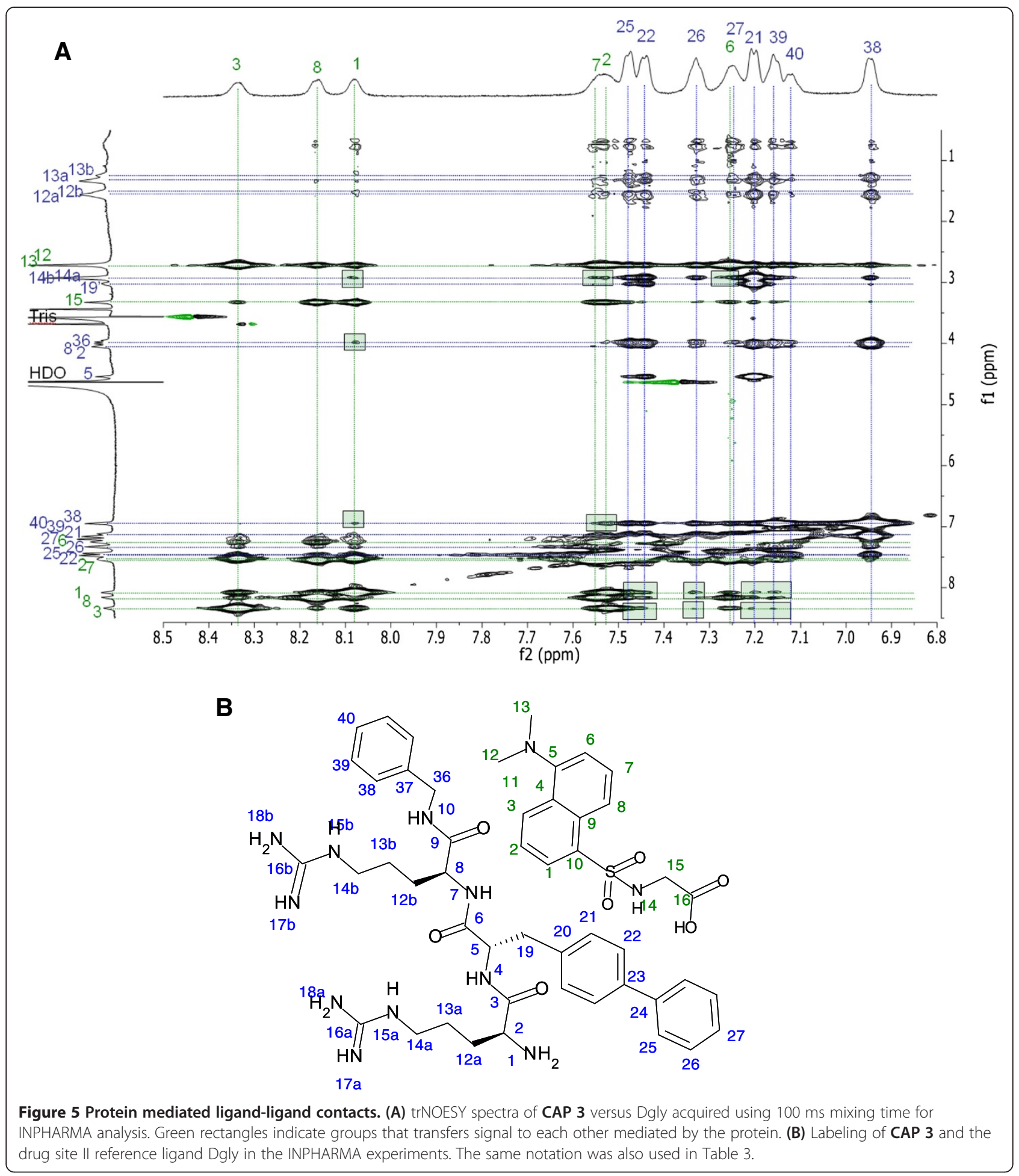

2XW0 structure, or towards Gln390 (2BXF). An intermediate position was observed for the apo structure 1E78. With Arg410 in 2XVQ resembling the 2XW0 conformation and the side chain in 2XW1 in a similar position as seen in $2 \mathrm{BXF}$. The apo structure 1E78 did not produce satisfactory poses for the system with a few exceptions, and comparing the docking score with the experimental binding data indicated a too weak interaction for these poses. Hence, when the side chain was positioned in the middle of the entrance of drug site II, Arg410 was blocking the binding site. Docking with 2XW0 and 2XVQ as target structures did not produce 
Table 3 NMR-INPHARMA results of CAP 3 versus Dgly

\begin{tabular}{cccccccccc}
\hline Entry & & \multicolumn{7}{c}{ Dgly } \\
\cline { 2 - 9 } & Label & $\mathbf{1}$ & $\mathbf{2}$ & $\mathbf{3}$ & $\mathbf{6}$ & $\mathbf{7}$ & $\mathbf{8}$ & $\mathbf{1 2 / 1 3}$ & $\mathbf{1 5}$ \\
\hline & 19 & + & - & - & $\mathrm{n} / \mathrm{a}$ & - & - & +++ & ++ \\
& 21 & + & $\mathrm{n} / \mathrm{a}$ & + & $\mathrm{n} / \mathrm{a}$ & $\mathrm{n} / \mathrm{a}$ & ++ & +++ & ++ \\
& 22 & ++ & $\mathrm{n} / \mathrm{a}$ & ++ & $\mathrm{n} / \mathrm{a}$ & $\mathrm{n} / \mathrm{a}$ & ++ & +++ & ++ \\
& 25 & ++ & $\mathrm{n} / \mathrm{a}$ & ++ & $\mathrm{n} / \mathrm{a}$ & $\mathrm{n} / \mathrm{a}$ & $\mathrm{n} / \mathrm{a}$ & +++ & ++ \\
& 26 & ++ & $\mathrm{n} / \mathrm{a}$ & ++ & $\mathrm{n} / \mathrm{a}$ & $\mathrm{n} / \mathrm{a}$ & - & +++ & + \\
& 27 & $\mathrm{n} / \mathrm{a}$ & $\mathrm{n} / \mathrm{a}$ & $\mathrm{n} / \mathrm{a}$ & $\mathrm{n} / \mathrm{a}$ & $\mathrm{n} / \mathrm{a}$ & $\mathrm{n} / \mathrm{a}$ & +++ & $\mathrm{n} / \mathrm{a}$ \\
& 36 & + & + & - & + & + & - & +++ & + \\
& 38 & - & - & - & ++ & ++ & ++ & +++ & + \\
& 39 & + & $\mathrm{n} / \mathrm{a}$ & + & $\mathrm{n} / \mathrm{a}$ & $\mathrm{n} / \mathrm{a}$ & ++ & +++ & ++ \\
& 40 & + & $\mathrm{n} / \mathrm{a}$ & + & $\mathrm{n} / \mathrm{a}$ & $\mathrm{n} / \mathrm{a}$ & ++ & +++ & + \\
\hline
\end{tabular}

,+++ , and +++ denotes transferred signals and strength between groups in the two ligands (see Figure 5 for labeling), - indicate no signal transferred

between groups and $n / a$ denotes not available signals.

satisfactory results, as no accepted poses for CAP 3 or CAP 4 were obtained. The Arg410 is pointing toward Glu492 in a similar conformation for these structures, which does not seem to be the optimal conformation for CAP binding.

For 2BXF and 2XW1, which had Arg410 conformations toward Gln390, all of the peptides and the reference ligands were satisfactorily docked and with scores in agreement with the experimental ITC data in Table 2. However, 2XW1 produced more numerous and consistent of accepted poses compared to 2BXF for all of the binding CAPs. 2XW1 was also able to rank the nonbonding control peptide CAP $\mathbf{1}$ at the bottom of the ranking list. As a consensus for the accepted poses for these targets, the peptides interact with drug site II by one of the hydrophobic moieties, either the indole for CAP 2 or the Bip side chain for CAP 3 and CAP 5 respectively. Interestingly, only the benzyl capping group residue was found to interact with drug site II for CAP 4. This is most likely due to the large size of the synthetic Tbt side chain in this peptide. Compared with the NMR INPHARMA results and the epitope mapping by others [37] the signal transferred between the different structural parts of Dgly and CAP 3 was in agreement with how deep they would be located in the binding site. For instance the signal from the glycine methylene group show indications of transferring to parts of the CAP 3 which would occupy the outer part of drug site II.

Figure 7 shows docking poses for the highest accepted ranked pose of CAP 3 and CAP 4 superimposed on the docked Dgly molecule for target structure 2XW1. The docking scores for the $2 \mathrm{XW} 1$ target were in the range of -6.6 to $-8.3 \mathrm{kcal} / \mathrm{mol}$ for the top ranked accepted pose of the CAPs and $-9.0 \mathrm{kcal} / \mathrm{mol}$ for Dgly, corresponding to lower $\mu \mathrm{M}$ binding constants. Whereas for the range for 2BXF was -5.7 to $-7.7 \mathrm{kcal} / \mathrm{mol}$ for the CAPs and $-8.0 \mathrm{kcal} / \mathrm{mol}$ for the top ranked pose of Dgly. The CAP 3 was top ranked for both targets with a score of -10.8 (2XW1) and $-9.0(2 \mathrm{BXF}) \mathrm{kcal} / \mathrm{mol}$ which correspond to a binding constant in lower $\mathrm{nM}$ region. In these two poses the Bip binds deeper in the binding site than any of the other poses for this peptide, and it is uncertain if it corresponds to a real binding mode when comparing with the experimental binding constant. In general the control peptide CAP 5 displayed docking poses that resembled the Bip conformation of CAP 3. Control peptide CAP 1 was given docking scores that correspond to mid $\mu \mathrm{M} K_{d}$ values, and docked poses were exclusively located outside drug site II for the targets 2BXF and 2XW1.

From the docking poses, the interaction with drug site II of HSA depends only on the hydrophobic elements of the peptides, and does not involve the cationic residues that are vital for antimicrobial activity. The models are in good agreement with the experimental NMR results in this aspect. This is also evident when comparing the experimental and modelled interaction of the two control peptides CAP 1 and CAP 5 with HSA. The control for the cationic part of the pharmacophore, CAP 1, does not bind to HSA. Whereas CAP $\mathbf{5}$ which is containing only hydrophobic residues interacts with drug site II with similar affinity as the antimicrobial CAPs. The arginines were observed to interact with nearby negatively charged surface residues in the docking poses, but no consistent patterns were observed. The solvent states of the arginine residues are considered equal in the bound and free form of the peptides, and will therefore not contribute to the free energy of the binding. It seems that the CAPs have evaded their own undesirable characteristics as drug site II ligands, as they only interact with one of their lipohilic groups.

Avoiding albumin binding for CAPs would be challenging, as the hydrophobic moieties are crucial for their antimicrobial activity. But observations in the docking poses of CAP 4 might be a starting point in changing the albumin binding properties. The bulky Tbt side chain of this CAP was observed to be too large to interact satisfactory with drug site II, and hence the interaction was achieved by binding with the C-terminal capping benzyl. If a lipophilic group with similar properties would replace the benzyl, lower albumin binding is anticipated.

\section{Crystallization}

The attempts to crystallize and obtain a complex structure of HSA and CAPs resulted in an apo-structure with fatty acid molecules bound in the seven fatty acid binding sites (Additional file 3), as previously described in the literature [40-42]. In our apo-fatty acid structure we found that for the first time to our knowledge, the direction of the fatty 


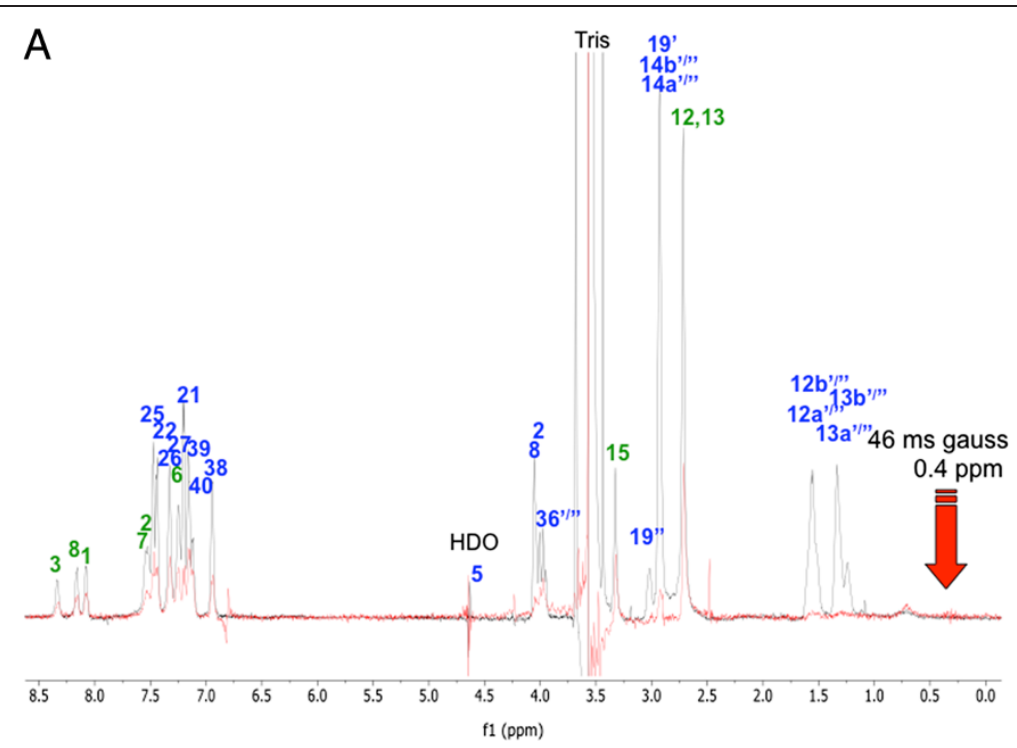

B
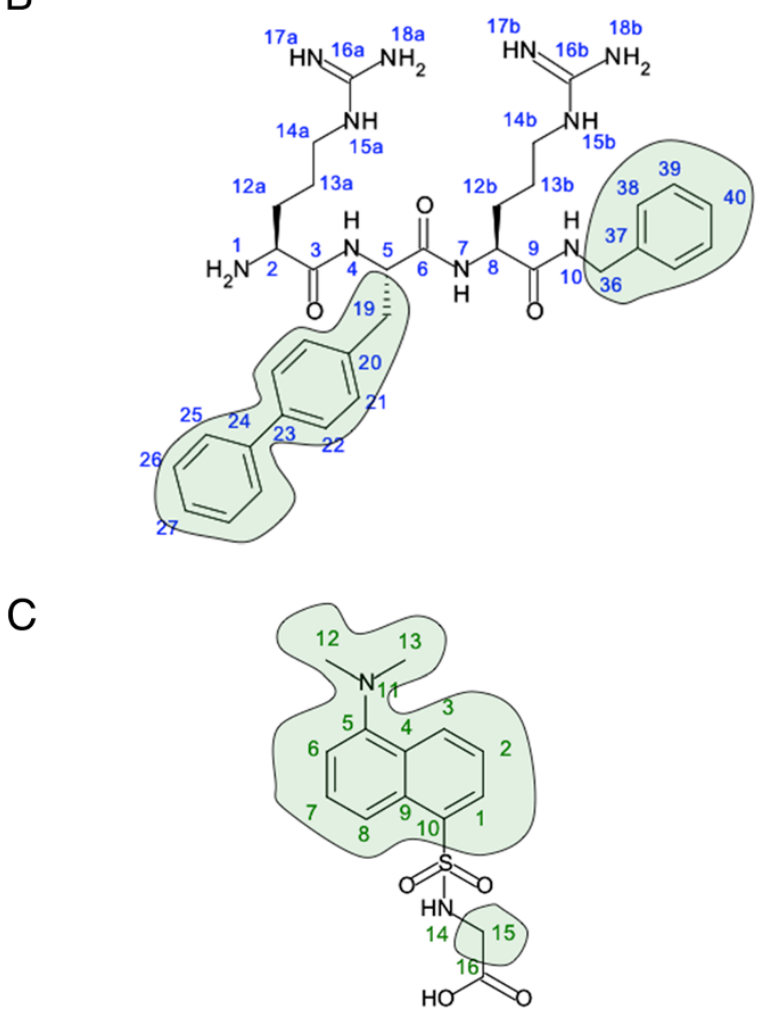

Figure 6 Protein contact mapping of ligands. (A) STD spectra of CAP 3, Dgly and HSA in 100:1 ratio (red) superimposed on a dpfgse proton spectra acquired with a $10 \mathrm{~ms}$ T2 filter for protein suppression (grey). In (B) the exited hydrophobic parts of CAP $\mathbf{3}$ are indicated in green, and in (C) the entire molecule of drug site II reference ligand Dgly was exited.

acid bound in FA7 is conclusively determined. The carboxyl group was found to form strong ionic interactions $(<3 \AA)$ with the guanidinium group of Arg218, see Additional file 3 Figure S8. Data collection and model refinement statistics are presented in Additional file 3: Table S1.

\section{Conclusions}

In this study we have reported how CAPs interact with HSA within $\mu \mathrm{M}$ affinity by ITC in agreement with previous studies by our group [21]. We have identified the binding site of the peptides to conclusively be drug site 


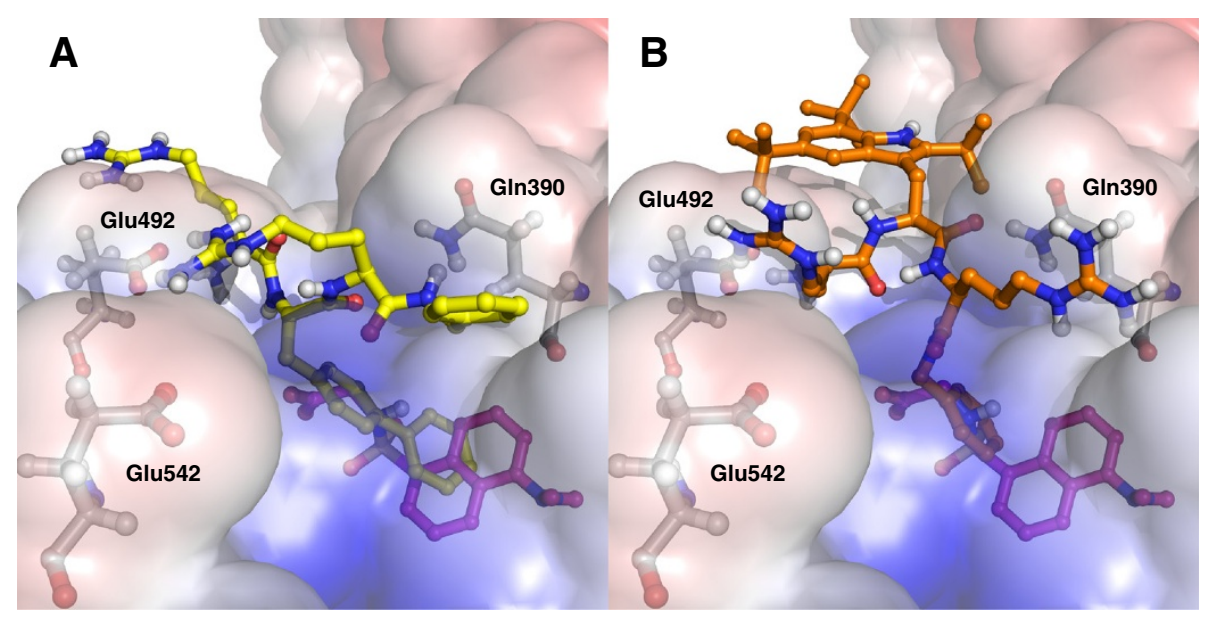

Figure 7 Molecular models of ligand binding to human albumin obtained with docking. (A) Docking poses of CAP $\mathbf{3}$ in yellow (docking score $-8.1 \mathrm{kcal} / \mathrm{mol}$ ) superimposed on Dgly in magenta (docking score $-9.0 \mathrm{kcal} / \mathrm{mol}$ ) with target $2 \mathrm{XW}$. (B) Docking poses of CAP 4 in orange (docking score $-6.6 \mathrm{kcal} / \mathrm{mol}$ ) superimposed on Dgly in magenta (docking score $-9.0 \mathrm{kcal} / \mathrm{mol}$ ) with target $2 \mathrm{XW} 1$. The calculated electrostatic potential surface of $2 X W 1$ is shown. CAP $\mathbf{3}$ is interacting with drug site II with the biphenyl, whereas CAP $\mathbf{4}$ is binding with the C-terminal capping benzyl. The lipophilic group in each peptide is comprising the volume occupied by the docked Dgly conformation. Arg410 is omitted in the figures merely for the purpose of clarification, as it would partly cover the bound Dgly and CAPs.

II of HSA. The interaction is solely dependent on the hydrophobic moieties present in the peptides. Both NMR experiments and molecular modelling results support that the cationic arginine residues of the peptides do not contribute to the interaction. Since the hydrophobic moieties are an important part of the pharmacophore of CAPs, it will be challenging to design peptides with satisfactory activity with reduced albumin binding properties, and hence it is anticipated that HSA binding of CAPs will be an issue that needs to be addressed in future drug administration strategies.

\section{Methods}

\section{Ligand library}

The molecular structures of the ligands used in this study are presented in Figure 1. The synthesis of CAP 2 [23], CAP 3 [21,23] and CAP 4 [24] have been published elsewhere by our group. The control peptides CAP 1 and CAP 5 were purchased from PolyPeptide Laboratories (Strasbourg, France). Reference ligands for drug site I warfarin (A4571) and for drug site II dansylglycine (D0875) were purchased from Sigma. CAP 2-4 have a common RXR-Bzl scaffold, with X containing a varying hydrophobic side chain. The side groups were indole (Trp) for CAP 2, biphenyl (Bip) for CAP 3 and tri-tert-butyl substituted indole (Tbt) for CAP 4. CAP 1 and CAP 5 were included as inactive controls representing the cationic charge or the hydrophilic moiety of the active peptides respectively. Control peptide CAP 1 contained a methyl group (Ala) as side chain in $\mathrm{X}$ that was flanked by arginines and had an amide capped C- terminal. The control peptide CAP $\mathbf{5}$ had a Bip in the side group for $\mathrm{X}$ flanked by alanines and also contained an amide capped $\mathrm{C}$-terminal. The peptides were chosen for this study based on the diversity in lipophilicity and size of the varying hydrophobic side chain in residue $\mathrm{X}$.

\section{Microbiological studies}

The antibacterial activity of CAP $\mathbf{1}$ and CAP 5 were tested towards Staphylococcus aureus strain ATCC 25923, methicillin resistant Staphylococcus aureus strain ATCC 33591 and Escherichia coli strain ATCC 25922. The studies were performed by Toslab AS employing standard methods [43].

\section{Isothermal titration calorimetry studies}

The experiments were either performed on a CSC 5300 Nano-Isothermal Titration Calorimeter III (Calorimetry Sciences Corporation, Utah, USA) with a cell volume of $1 \mathrm{~mL}$, or a MicroCal ${ }^{\mathrm{m}} \mathrm{iTC}_{200}$ with a cell volume of $200 \mu \mathrm{L}$ (MicroCal, LLC., Northampton, MA, USA). In all experiments lyophilized HSA $29 \%$ essential fatty acid and globulin free (Sigma A3782), was weighed out to a nominal concentration and dissolved prior to the experiments in buffer $50 \mathrm{mM}$ Tris, $10 \mathrm{mM} \mathrm{CaCl}_{2}$, $\mathrm{pH} 7.4$ at $25^{\circ} \mathrm{C}$. All ligands and peptides were dissolved in the same buffer. Control titrations of ligand into buffer were performed in duplicate to investigate if the heat of dilution would generate a significant signal, and if ligand-ligand interactions were present in the competitive experiments. The same settings as for the 
corresponding ligand-HSA experiments were applied in the control experiments.

In all experiments applying the CSC 5300 NanoIsothermal Titration Calorimeter III, the HSA concentration was $0.1 \mathrm{mM}$ and all other peptides and ligand concentrations used were $2.1 \mathrm{mM}$. In the non-competitive design 33 consecutive $3 \mu \mathrm{L}$ injections were carried out with $200 \mathrm{~s}$ spacing between each injection. A stirring rate of $150 \mathrm{rpm}$ and an isotherm temperature of $25^{\circ} \mathrm{C}$ were applied. The response signal was measured at $1 \mathrm{~s}$ intervals, and a $200 \mathrm{~s}$ baseline was collected prior to the first injection for the purpose of assessing the baseline. Noncompetitive experiments were carried out either in duplicates or triplicates. In the competitive experimental design HSA was pre-incubated with either the drug site I warfarin or site II dansylglycin ligands, or the peptides CAP 2-4 in a 1:1 molar ratio. In total 40 consecutive injections were performed with a volume of $5 \mu \mathrm{L}$ and a spacing of $300 \mathrm{~s}$. The first injection in the series had a dummy volume of $3 \mu \mathrm{L}$ to account for leakage from the syringe and was omitted in the final analysis. Prior to the first injection, a baseline of $100 \mathrm{~s}$ was recorded to ensure instrument stability. The solution was stirred at $150 \mathrm{rpm}$, and the isothermal temperature set to $25^{\circ} \mathrm{C}$. All competitive experiments were carried out in duplicate. For the experiments conducted on the MicroCal ${ }^{\mathrm{Tm}} \mathrm{iTC}_{200}$ instrument the HSA concentration used was $0.21 \mathrm{mM}$, and the concentration of ligands and peptides were $4.3 \mathrm{mM}$. In total, 29 injections of $1.35 \mu \mathrm{L}$ with $180 \mathrm{~s}$ spacing between each injection were titrated. The first injection was set to a dummy volume of $0.1 \mu \mathrm{L}$ due to syringe leakage in the baseline equilibration step. An initial delay of $180 \mathrm{~s}$ was set to sample the baseline. The applied reference power was $6 \mu \mathrm{cal} / \mathrm{s}$ and an isothermal temperature of $25^{\circ} \mathrm{C}$ was used. The solution was stirred at $1000 \mathrm{rpm}$, applying high feedback mode and a filter period of $5 \mathrm{~s}$. For competitive experiments HSA was pre-incubated with the reference ligand or peptide at a molar ratio of 1:3. For the selected peptides CAP 3 and CAP 5 competitive experiments with reversed order of the reference ligands and the CAP were performed as a control of titration order. The same ITC settings were applied for the noncompetitive and competitive experiments with the MicroCal $^{\text {Th }} \mathrm{iTC}_{200}$.

The ITC data was analyzed using the NanoAnalyse software from TA Instruments, v2.0.1 (Waters LLC, New Castle, DE, US) or Origin ${ }^{\circ}$ 7.0. Independent model fit was used to generate the binding isotherm, and the first injection in all titration experiments was omitted in the binding isotherm analysis. The heat of dilution and unspecific binding signal corresponding to the last injection in each experiment was subtracted from all of the heat signals. The quality of the model fit was examined using the statistical modules of the software, the $95 \%$ confidence interval based on a 1000 trial calculations in the NanoAnalyze package, and the default non-weighted least square calculation in Origin ${ }^{\circ}$ 7.0.

\section{NMR}

All NMR experiments were acquired on an Agilent (Varian) inova spectrometer operating at $599.934 \mathrm{MHz}$ for ${ }^{1} \mathrm{H}$, equipped with a $2^{\text {nd }}$ generation inverse triple resonance HCN cold probe. NMR samples were prepared in $50 \mathrm{mM}$ Tris buffer, $10 \mathrm{mM} \mathrm{CaCl}_{2}, \mathrm{pH} 7.4$ at $25^{\circ} \mathrm{C}$, to final concentrations of $40 \mu \mathrm{M}$ HSA and $800 \mu \mathrm{M}$ ligand (Wrf, Dgly, CAP 1, CAP 3 and CAP 5). 1D-NOE ePHOGSY for ligand detection via WaterLOGSY $[36,44]$ was acquired as 256 transients, $8 \mathrm{k}$ complex points and $12000 \mathrm{~Hz}$ sweep width using $1500 \mathrm{~ms}$ mixing time, $1.0 \mathrm{~s}$ relaxation delay and a solvent selective pulse of $2.4 \mathrm{~ms}$ width at $12 \mathrm{~dB}$. Each ligand addition was recorded with standard ${ }^{1} \mathrm{H}$ spectra using watergate (3919) solvent suppression and blank controls were acquired before the addition of HSA samples for STD spectra [45] and transferred NOE (trNOE) spectra for INPHARMA-type analysis [46] were prepared in deuterated buffer to final concentrations of $10 \mu \mathrm{M}$ HSA and $1 \mathrm{mM}$ ligand (1:100). STD spectra were acquired in 256 transients, $6 \mathrm{k}$ complex data points and $12000 \mathrm{~Hz}$ sweep width. Saturation was achieved by 50 cycles of $45.8 \mathrm{~ms}$ gaussian shaped saturation pulses centered at $0.4 \mathrm{ppm}$ for the "on" resonance and at $15 \mathrm{ppm}$ for the "off" resonance fid. The difference spectra were produced by internal subtraction and all spectra were acquired with both sculpted solvent suppression during the PFG spin echo and $500 \mathrm{~ms}$ solvent presaturation during the relaxation delay. The protein signals were suppressed by a $10 \mathrm{~ms}$ T1 $\rho$-spinlock. Finally, the spectra were multiplied with a $3 \mathrm{~Hz}$ exponential window function. For reference, double PFG spin echo 1D proton spectra were acquired using the same parameters, including the $10 \mathrm{~ms}$ T1pspinlock for protein suppression. NOESY spectra for INPHARMA were acquired in 8 transients as $1440 \times 256$ data points at mixing times of 50,100, 200 and $500 \mathrm{~ms}$ using zero quantum (ZQ) filter and grad-90-grad randomization. All samples were monitored for line broadening, quickened relaxation and NOE inversion to spot any direct interaction between ligands before the addition of HSA.

\section{Crystallization studies}

An attempt was made to obtain crystals of the human albumin CAP complex for structural determination. Crystallization trials were carried out with recombinant human albumin (rHA) using both the soaking method with fatty acid rHA complex and co-crystallization methods as described by the Curry group [38,40]. Only the soaked fatty acid rHA complex produced crystals of 
satisfactory diffraction quality. The experimental details for the crystallization of rHA in complex with fatty acid are provided in the Additional file 3.

\section{Molecular docking}

Targets used in the molecular docking experiments were the apo HSA crystal structure 1E78 [47], and the four complex structures 2BXF [38], 2XW1 [3], 2XVQ [3], and 2XW0 [3]. The peptide library was built and prepared in LigPrep, version 2.5 [48], with their stereochemistries retained as all L-residues. All receptor targets were processed using the Protein Preparation Wizard in Maestro, version 9.3 [48]. The docking grids set up in Glide, version 5.8 [48], were centred either on the ligand in the complex structure, or one of the residues belonging to drug site II for the apo structure. A maximum size of ligand diameter and a $14 \AA$ mid point box were utilized. For the docking experiments the standard precision (SP) mode was applied. The grid generation and docking procedure was repeated due to the size difference of the smaller reference ligands and the larger CAPs, applying CAP 3 as reference ligand for the box size. The ligand library was comprised of the CAPs and the reference ligands Wrf and Dgly presented in Figure 1. For the targets 2XW1 and 2XVQ the side chain coordinates of Arg410 were not reported, and the residue was therefore built and minimized by Prime, version 3.1 [48].

\section{Additional files}

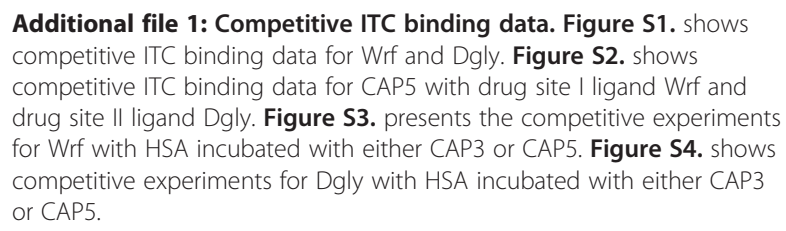

Additional file 2: WaterLOGSY experiments. Figure S5. presents WaterLOGSY of HSA and Wrf, Dgly and CAP 1, while Figure S6. shows WaterLOGSY of HSA and CAP1, CAP3 and CAP5.

Additional file 3: Crystal structure of albumin-palmitic acid complex. Crystallization conditions and details of data collection as refinement procedures are presented. A brief discussion of the crystal structure is provided along with data collection and refinement statitistics (Table S1). Figure S7. shows the mount crystal, and Figure $\mathbf{8 8}$. presents the electron density for the fatty acid binding mode of PA 7 in FA site 7.

\section{Abbreviations}

ADMET: Administration, distribution, metabolism, excretion and toxicity; AMP: Antimicrobial peptide; Bip: Biphenyl; CAP: Synthetic cationic antimicrobial peptide; Dgly: Dansylglycine; FA: Fatty acid binding site; GEM: Group epitope mapping; HSA: Human serum albumin; INPHARMA: Inter-ligand NOE for pharmacophore mapping; ITC: Isothermal titration calorimetry; MD: Molecular dynamics; MIC: Minimal inhibitory concentration; SAR: Structure-activity relationship; STD: Saturation transfer difference; Tbt: Tri-tert-butyl tryptophan; Wrf: Warfarin; WaterLOGSY: Water ligand observation with gradient spectroscopy.

\section{Competing interests}

The authors declare that they have no competing interests.

\section{Authors' contributions}

AS carried out the ITC experiments, crystallization, molecular docking and drafted the manuscript. Jl carried out of the NMR experiments, and contributed to the data interpretation. HKSL contributed to the data analysis. JS and JSS participated to the conception and in the design of the study. BOB conceived the study, participated in its design and helped to draft the manuscript. All authors read and approved the final manuscript.

\section{Acknowledgements}

The authors gratefully acknowledge Wenche Stensen for providing CAP 2-4. This work has been supported by the Research Council of Norway through a Centre of Excellence Grant (Grant No. 179568/N30).

\section{Author details}

${ }^{1}$ The Norwegian Structural Biology Centre, Department of Chemistry, Faculty of Science and Technology, University of Tromsø, NO-9037 Tromsø, Norway. ${ }^{2}$ Drug Discovery and Design, Department of Chemistry, Faculty of Science and Technology, University of Tromsø, NO-9037 Tromsø, Norway.

${ }^{3}$ Department of Chemistry, Faculty of Science and Technology, University of Tromsø, NO-9037 Tromsø, Norway. ${ }^{4}$ Centre for Theoretical and Computational Chemistry, Department of Chemistry, Faculty of Science and Technology, University of Tromsø, NO-9037 Tromsø, Norway.

Received: 24 April 2013 Accepted: 17 January 2014

Published: 23 January 2014

\section{References}

1. Kragh-Hansen U: Molecular aspects of ligand binding to serum albumin Pharmacol Rev 1981, 33(1):17-53.

2. He XM, Carter DC: Atomic structure and chemistry of human serum albumin. Nature 1992, 358(6383):209-215.

3. Ryan AJ, Ghuman J, Zunszain PA, Chung C-W, Curry S: Structural basis of binding of fluorescent, site-specific dansylated amino acids to human serum albumin. J Struct Biol 2011, 174(1):84-91.

4. Hein KL, Kragh-Hansen U, Morth JP, Jeppesen MD, Otzen D, Møller JV, Nissen P: Crystallographic analysis reveals a unique lidocaine binding site on human serum albumin. J Struct Biol 2010, 171(3):353-360.

5. Kremer JM, Wilting J, Janssen LH: Drug binding to human alpha-1-acid glycoprotein in health and disease. Pharmacol Rev 1988, 40(1):1-47.

6. Grandison MK, Boudinot FD: Age-related changes in protein binding of drugs: implications for therapy. Clin Pharmacokinet 2000, 38(3):271-290.

7. Fasano M, Curry S, Terreno E, Galliano M, Fanali G, Narciso P, Notari S, Ascenzi $P$ : The extraordinary ligand binding properties of human serum albumin. IUBMB Life 2005, 57(12):787-796.

8. Zasloff M: Antimicrobial peptides of multicellular organisms. Nature 2002, 415(6870):389-395.

9. Hancock REW, Sahl H-G: Antimicrobial and host-defense peptides as new anti-infective therapeutic strategies. Nat Biotechnol 2006, 24(12):1551-1557.

10. Boman HG: Peptide antibiotics and their role in innate immunity. Annu Rev Immunol 1995, 13:61-92.

11. Diamond G: Natures antibiotics: the potential of antimicrobial peptides as new drugs. Biologist 2001, 48(5):209-212.

12. Shai Y: Mode of action of membrane active antimicrobial peptides. Biopolymers 2002, 66(4):236-248.

13. Huang HW: Action of antimicrobial peptides: two-state model. Biochemistry 2000, 39(29):8347-8352

14. Bellamy W, Takase M, Yamauchi K, Wakabayashi H, Kawase K, Tomita M: Identification of the bactericidal domain of lactoferrin. Biochim Biophys Acta 1992, 1121(1-2):130-136.

15. Rekdal $\varnothing$, Andersen J, Vorland LH, Svendsen JS: Construction and synthesis of lactoferricin derivatives with enhanced antibacterial activity. J Pept Sci 1999, 5:32-45

16. Strøm MB, Haug BE, Skar ML, Stensen W, Stiberg T, Svendsen JS: The pharmacophore of short cationic antibacterial peptides. J Med Chem 2003, 46(9):1567-1570.

17. Haug BE, Stensen W, Stiberg T, Svendsen JS: Bulky nonproteinogenic amino acids permit the design of very small and effective cationic antibacterial peptides. J Med Chem 2004, 47(17):4159-4162.

18. Svenson J, Karstad R, Flaten GE, Brandsdal B-O, Brandl M, Svendsen JS: Altered activity and physicochemical properties of short cationic 
antimicrobial peptides by incorporation of arginine analogues. Mol Pharm 2009, 6(3):996-1005.

19. Karstad R, Isaksen G, Wynendaele E, Guttormsen Y, De Spiegeleer B, Brandsdal B-O, Svendsen JS, Svenson J: Targeting the S1 and S3 subsite of trypsin with unnatural cationic amino acids generates antimicrobial peptides with potential for oral administration. J Med Chem 2012 55(14):6294-6305.

20. Karstad R, Isaksen G, Brandsdal B-O, Svendsen JS, Svenson J: Unnatural amino acid side chains as S1, S1', and S2' probes yield cationic antimicrobial peptides with stability toward chymotryptic degradation. J Med Chem 2010, 53(15):5558-5566.

21. Svenson J, Brandsdal B-O, Stensen W, Svendsen JS: Albumin binding of short cationic antimicrobial micropeptides and its influence on the in vitro bactericidal effect. J Med Chem 2007, 50(14):3334-3339.

22. Findlay B, Zhanel GG, Schweizer F: Investigating the antimicrobial peptide 'window of activity' using cationic lipopeptides with hydrocarbon and fluorinated tails. Int J Antimicrob Agents 2012, 40(1):36-42

23. Svenson J, Stensen W, Brandsdal B-O, Haug BE, Monrad J, Svendsen JS: Antimicrobial peptides with stability toward tryptic degradation. Biochemistry 2008, 47(12):3777-3788.

24. Haug BE, Stensen W, Kalaaji M, Rekdal $\varnothing$, Svendsen JS: Synthetic antimicrobial peptidomimetics with therapeutic potential. J Med Chem 2008, 51(14):4306-4314.

25. Svenson J, Vergote V, Karstad R, Burvenich C, Svendsen JS, De Spiegeleer B: Metabolic fate of lactoferricin-based antimicrobial peptides: effect of truncation and incorporation of amino acid analogs on the in vitro metabolic stability. J Pharmacol Exp Ther 2010, 332(3):1032-1039.

26. Haug BE, Strøm MB, Svendsen JSM: The medicinal chemistry of short lactoferricin-based antibacterial peptides. Curr Med Chem 2007, 14(1):1-18.

27. Isaksson J, Brandsdal BO, Engqvist M, Flaten GE, Svendsen JSM, Stensen W: A synthetic antimicrobial peptidomimetic (LTX 109): stereochemical impact on membrane disruption. J Med Chem 2011, 54(16):5786-5795.

28. Liu Z, Deshazer H, Rice AJ, Chen K, Zhou C, Kallenbach NR: Multivalent antimicrobial peptides from a reactive polymer scaffold. J Med Chem 2006, 49(12):3436-3439.

29. Liu Z, Brady A, Young A, Rasimick B, Chen K, Zhou C, Kallenbach NR: Length effects in antimicrobial peptides of the (RW)n series. Antimicrob Agents Chemother 2007, 51(2):597-603.

30. Liu Z, Young AW, Hu P, Rice AJ, Zhou C, Zhang Y, Kallenbach NR: Tuning the membrane selectivity of antimicrobial peptides by using multivalent design. ChemBioChem 2007, 8(17):2063-2065.

31. Loun B, Hage DS: Chiral separation mechanisms in protein-based HPLC columns. 1. Thermodynamic studies of (R)- and (S)-warfarin binding to immobilized human serum albumin. Anal Chem 1994, 66(21):3814-3822

32. Loun B, Hage DS: Chiral separation mechanisms in protein-based HPLC columns. 2. Kinetic studies of (R)- and (S)-warfarin binding to immobilized human serum albumin. Anal Chem 1996, 68(7):1218-1225.

33. Abou-Khalil R, Jraij A, Magdalou J, Ouaini N, Tome D, Greige-Gerges H: Interaction of cucurbitacins with human serum albumin: thermodynamic characteristics and influence on the binding of site specific ligands. J Photochem Photobiol B Biol 2009, 95(3):189-195.

34. Sudlow G, Birkett DJ, Wade DN: The characterization of two specific drug binding sites on human serum albumin. Mol Pharmacol 1975 11(6):824-832

35. Muller N, Lapicque F, Drelon E, Netter P: Binding sites of fluorescent probes on human serum albumin. J Pharm Pharmacol 1994, 46(4):300-304

36. Dalvit C, Fogliatto G, Stewart A, Veronesi M, Stockman B: WaterLOGSY as a method for primary NMR screening: practical aspects and range of applicability. J Biomol NMR 2001, 21(4):349-359.

37. Lucas $L H$, Price $K E$, Larive CK: Epitope mapping and competitive binding of HSA drug site II ligands by NMR diffusion measurements. J Am Chem Soc 2004, 126(43):14258-14266

38. Ghuman J, Zunszain PA, Petitpas I, Bhattacharya AA, Otagiri M, Curry S: Structural basis of the drug-binding specificity of human serum albumin. J Mol Biol 2005, 353(1):38-52.

39. Curry S: Lessons from the crystallographic analysis of small molecule binding to human serum albumin. Drug Metab Pharmacokinet 2009, 24(4):342-357.
40. Curry S, Mandelkow H, Brick P, Franks N: Crystal structure of human serum albumin complexed with fatty acid reveals an asymmetric distribution of binding sites. Nat Struct Biol 1998, 5(9):827-835.

41. Bhattacharya AA, Grüne T, Curry S: Crystallographic analysis reveals common modes of binding of medium and long-chain fatty acids to human serum albumin. J Mol Biol 2000, 303(5):721-732.

42. Petitpas I, Grüne T, Bhattacharya AA, Curry S: Crystal structures of human serum albumin complexed with monounsaturated and polyunsaturated fatty acids. J Mol Biol 2001, 314(5):955-960.

43. Amsterdam D: Susceptibility testing of antimicrobials in liquid media. In Antibiotics in Laboratory Medicine. 4th edition. Edited by Lorian V. Baltimore, MD: Williams and Wilkins; 1996:75-78.

44. Dalvit $C$, Pevarello $P$, Tatò $M$, Veronesi M, Vulpetti $A$, Sundström $M$ : Identification of compounds with binding affinity to proteins via magnetization transfer from bulk water*. J Biomol NMR 2000, 18(1):65-68.

45. Mayer M, Meyer B: Group epitope mapping by saturation transfer difference NMR to identify segments of a ligand in direct contact with a protein receptor. J Am Chem Soc 2001, 123(25):6108-6117.

46. Sánchez-Pedregal VM, Reese M, Meiler J, Blommers MJJ, Griesinger C, Carlomagno T: The INPHARMA method: protein-mediated interligand NOEs for pharmacophore mapping. Angew Chem Int Ed Engl 2005 44(27):4172-4175

47. Bhattacharya AA, Curry S, Franks NP: Binding of the general anesthetics propofol and halothane to human serum albumin. High resolution crystal structures. J Biol Chem 2000, 275(49):38731-38738.

48. Schrödinger LLC: New York; 2012

doi:10.1186/1472-6807-14-4

Cite this article as: Sivertsen et al: Synthetic cationic antimicrobial peptides bind with their hydrophobic parts to drug site II of human serum albumin. BMC Structural Biology 2014 14:4.

\section{Submit your next manuscript to BioMed Central and take full advantage of:}

- Convenient online submission

- Thorough peer review

- No space constraints or color figure charges

- Immediate publication on acceptance

- Inclusion in PubMed, CAS, Scopus and Google Scholar

- Research which is freely available for redistribution 